\title{
Factores pronóstico asociados a la duración de la urticaria espontánea crónica en población colombiana
}

Prognostic factors associated with the duration of chronic spontaneous urticaria in a Colombian population

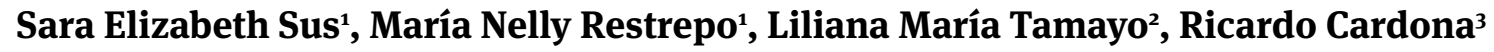 \\ 1. Médica, residente de Alergología Clínica, Universidad de Antioquia, Medellín, Colombia \\ 2. Médica dermatóloga, alergóloga clínica; docente de posgrado de Alergología Clínica, Universidad de Antioquia; docente de posgrado de \\ Dermatología, Universidad Pontificia Bolivariana, Medellín, Colombia \\ 3. Médico, magíster en Inmunología, alergólogo clínico; docente de posgrado de Alergología, Universidad de Antioquia, Medellín, Colombia
}

\section{Resumen}

INTRODUCCIón. No existen, hasta la fecha, marcadores clínicos o de laboratorio que permitan predecir la duración de la urticaria, con resultados reproducibles en los diferentes estudios realizados.

OBJETIVo. Explorar la asociación entre los factores de pronóstico y la duración de la urticaria espontánea crónica.

Metodología. Es un estudio retrospectivo en el que se exploran los factores asociados a la duración de la urticaria espontánea crónica en la población remitida al Servicio de Alergología Clínica de la IPS universitaria de la Universidad de Antioquia.

RESUltados. Se incluyeron 99 casos (67 mujeres y 32 hombres), con un promedio de edad de 40,3 años. El $71,7 \%$ presentó prueba positiva de plasma y suero autólogo. El 17,2 \% presentaba alteración tiroidea determinada por alteración de la tirotropina (TSH), presencia de anticuerpos antitiroideos o ambos. El $65,7 \%$ recibía tratamiento con antihistamínicos $\mathrm{H} 1$, el 17,2 \%, antihistamínico H1 más antagonista del receptor de leucotrienos, el 11,1\%, antihistamínicos $\mathrm{H} 1$ más antihistamínicos $\mathrm{H} 2$, y solo el $2 \%$ recibió tratamiento con medicamento inmunomodulador. La duración de la enfermedad mayor de 60 meses se asoció con angioedema, otros tipos de urticaria, enfermedades alérgicas cutáneas y sexo femenino.

Conclusiones. En la exploración de los factores asociados a la duración, aunque las variables estudiadas no fueron estadísticamente significativas, se observaron diferencias mayores del $10 \%$ que marcan una tendencia que pudiera ser significativa con un mayor tamaño de muestra, para las variables sexo femenino, presencia de angioedema, presencia de alergias en piel y la asociación con otros tipos de urticaria, lo cual concuerda con lo descrito en la literatura científica. La prueba de plasma y suero autólogo en nuestra cohorte no mostró tener ninguna asociación con la duración de la enfermedad.

Palabras Clave: urticaria, angioedema, prueba cutánea, pronóstico, urticaria/tratamiento, enfermedades tiroideas.

\section{Summary}

BACKGROUND: To date there are no clinical or laboratory markers to predict the duration of urticaria, with reproducible results among the different populations studied.

\section{Correspondencia:}

Sara Elizabeth Sus

\section{Email:}

sara.sus.carrizosa@hotmail.com

Recibido: 19 de noviembre de 2012. Aceptado: 15 de marzo de 2013.

No se reportan conflictos de intereses. 
OBJECTIVE: To explore the association between prognostic factors and duration of spontaneous chronic urticaria.

METHODOLOGY: We performed a retrospective study that explored factors associated with the duration of spontaneous chronic urticaria, at the Servicio de Alergología Clínica of the Universidad de Antioquia.

RESUltS: Ninety nine cases (67 women, 32 men) were studied, with a mean age of 40.3 years. Seventy one percent of patients tested positive for autologous serum-plasma skin test; $\mathbf{1 7 . 2} \%$ had thyroid impairment determined by TSH and/ or the presence of antithyroid antibodies; $65,7 \%$ received treatment with $\mathrm{H} 1$ antihistamines, $17.2 \% \mathrm{H} 1$-antihistamine and a leukotriene receptor antagonist, 11.1\% $\mathrm{H} 1$-antihistamines plus $\mathrm{H}_{2}$-antihistamines, and only $2 \%$ were treated with immunomodulatory drugs. The duration of the disease over 60 months was associated with angioedema, other types of urticaria, allergic skin diseases and female gender.

ConCLUSIONS: After exploring the factors associated with the persistence of spontaneous chronic urticaria, although the variables studied showed no statistically significant differences, there were differences in percentages greater than $10 \%$ for the variables sex, presence of angioedema, presence of skin allergies and association with other types of urticaria, marking a trend that could be significant with a larger sample size. Our findings are consistent with those described in the literature. The autologous serum-plasma skin test did not have any association with the duration of spontaneous chronic urticaria in our cohort.

KEY WORDS: Urticaria, angioedema, skin test, prognosis, urticaria/ treatment, thyroid diseases.

\section{Introducción}

La urticaria es una causa común de consulta médica, su diagnóstico es fundamentalmente clínico y se caracteriza por la presentación súbita de habones, angioedema o ambos ${ }^{1}$. La urticaria se clasifica en urticaria espontánea, urticaria física y otros tipos de urticaria. La urticaria espontánea se clasifica en urticaria aguda y urticaria crónica ${ }^{2}$ según la duración; la aguda tiene una duración menor de seis semanas y la crónica, una mayor de seis semanas3. Esta última tiene una prevalencia general de 0,5 a $1 \%$, 4.5 , puede presentarse a cualquier edad, con un pico de presentación entre los $20 \mathrm{y}$ los 40 años, y afecta principalmente a pacientes en edad productiva, con predominio en las mujeres ${ }^{6-8}$. El curso clínico de la urticaria crónica es impredecible y puede persistir durante unos meses ${ }^{4}$ hasta más de 10 años ${ }^{9}$, lo que dificulta su pronóstico y conlleva a un gran deterioro en la calidad de vida ${ }^{10,11}$.

En diferentes estudios se ha explorado la relación entre la duración, la intensidad y la respuesta al tratamiento en pacientes con urticaria crónica, con la presencia de otros tipos de urticaria, entre ellas las físicas ${ }^{9}$, paráme- tros de laboratorio como complemento, hemoleucograma, anticuerpos antinucleares, anticuerpos antitiroideos y niveles de IgE total ${ }^{12}$, presencia de angioedema ${ }^{13,14}$, hipertensión arterial y relación con enfermedades atópicas, con resultados que han sido controvertidos.

Teniendo en cuenta los hallazgos reportados hasta la fecha en relación con el curso natural y a los factores de pronóstico, los datos actuales son contradictorios y la mayoría no se presentan en términos de odds ratios ni riesgos relativos. En Latinoamérica existen reportes de estudios descriptivos que evalúan las características y la evolución natural de la urticaria ${ }^{15}$, pero ninguno ha explorado los factores asociados a la duración o la intensidad.

\section{Metodología}

Se trató de un estudio de cohorte con análisis retrospectivo, llevado a cabo mediante la revisión de las historias clínicas de los participantes y, también, del registro de la prueba de suero y plasma autólogo.

Se incluyeron todos los pacientes con diagnóstico 
de urticaria espontánea crónica, que acudieron al Servicio de Alergología Clínica de la Universidad de Antioquia-IPS universitaria, sede ambulatoria, en el periodo comprendido entre enero de 2006 y febrero de 2011, a quienes se les practicó la prueba de plasma y suero autólogo, y que continuaron su control médico en dicha institución.

El diagnóstico de urticaria crónica fue clínico, definido por la presencia de habones, angioedema o ambos, de duración mayor de seis semanas sin un factor desencadenante claro, los cuales ocurrían la mayoría de los días de la semana; el diagnóstico se basó en el criterio médico de un especialista en alergología.

En el Servicio de Alergología Clínica se elabora de forma rutinaria una anamnesis dirigida a pacientes con urticaria crónica, en la que se interroga sobre el uso de medicamentos concomitantes, factores agravantes o atenuantes de los sintomas, antecedentes familiares y personales de atopia, autoinmunidad, presencia de otras enfermedades sistémicas, en especial hipertensión arterial o enfermedad tiroidea, y necesidad de medicamentos para controlar sus síntomas. También, se determina rutinariamente la actividad de la enfermedad en: ausente, leve, moderara o intensa, de acuerdo con el sistema unificado de puntaje de actividad de la urticaria propuesto por la European Academy of Allergy and Clinical Immunology (EAACI), la Global Allergy and Asthma European Network (GA2LEN), European Dermatology Forum (EDF) y la Organización Mundial de Alergias (WAO) en las guías sobre definición, clasificación y diagnóstico de urticaria, el cual se basa en la presencia de habones y prurito en una escala de puntuación de o a $6^{16}$.

\section{Criterios de inclusión}

Se incluyeron los pacientes que tuvieran diagnóstico de urticaria espontánea crónica, que acudieron al Servicio de Alergología Clínica de la Universidad de Antioquia-IPS universitaria, sede ambulatoria, en el periodo comprendido entre enero de 2006 y febrero de 2011, a quienes se les practicó prueba de plasma y suero autólogo, y que continuaron su control médico en dicha institución.

\section{Criterios de exclusión}

Se excluyeron los pacientes con diagnóstico de urticaria vasculítica demostrada por biopsia, angioedema hereditario o adquirido y urticaria aguda, y los que acudieron al Servicio de Alergología Clínica de la Universidad de Antioquia-IPS universitaria, sede ambulatoria, solo para practicarse la prueba de suero autólogo y no continuaron su seguimiento en dicha institución.

A todos los se les prescribió, como parte del protocolo de tratamiento de la urticaria crónica, una orden de desparasitación dada la alta asociación descrita entre la urticaria crónica y la presencia de parasitosis gastrointestinal ${ }^{17}$. Se prescribió tinidazol ( $2 \mathrm{~g}$ cada 24 horas durante dos días), albendazol (400 mg, en dosis única) y teclozán (500 mg cada 12 horas por tres dosis). En la población pediátrica se ajustó la dosis según el peso, de la siguiente manera: tinidazol, $50 \mathrm{mg} / \mathrm{kg}$ por día durante dos días, y albendazol, $400 \mathrm{mg}$ en dosis única.

A todos los pacientes se les ordenó hemoleucograma, valoración de tirotropina (TSH), determinación de anticuerpos antimicrosómicos y antiperoxidasa, endoscopia de vías digestivas en aquellos con síntomas gastrointestinales y prueba positiva de plasma y suero autólogo².

\section{Prueba de suero autólogo}

La prueba de plasma y suero autólogo se practicó de la siguiente forma: todos los pacientes debian haber suspendido el consumo de medicamentos antihistamínicos como mínimo siete días antes de la prueba, y aquellos que recibían tratamiento con doxepina o con antidepresivos tricícliclos, los debían suspender dos semanas antes de la prueba. Los que recibían glucocorticoides orales en dosis menor de $15 \mathrm{mg}$ por día, no requirieron suspender el tratamiento; ningún paciente recibió tratamiento a dosis mayores de esta. Ninguno se encontraba en tratamiento con otro tipo de inmunomoduladores, como ciclosporina, azatioprina o micofenolato, al momento de la prueba.

Mediante punción de la vena antecubital con aguja vacuitainer (Becton, Dickenson and Company, Franklin Lakes, NJ, USA), se recolectó sangre en dos tubos, $4 \mathrm{ml}$ en el tubo de tapa lila con EDTA BD Vacutainer ${ }^{\circledR}$, para la extracción de plasma, y $7 \mathrm{ml}$ en el tubo de tapa roja sin anticoagulante BD Vacutainer ${ }^{\circledR}$, para la extracción de suero. El tubo sin anticoagulante se dejó a temperatura ambiente por 30 minutos para permitir la formación del coágulo. Posteriormente, se centrifugaron los dos tubos a 3.500 revoluciones por minuto, el de tapa roja por 18 minutos y el de tapa lila por 10 minutos, para obtener el suero y el plasma para la prueba.

La prueba se practicó sobre la región volar del brazo luego de hacer una adecuada técnica de limpieza con antiséptico tópico, evitando el área cercana a la muñeca o al pliegue antecubital. Se utilizó jeringa estéril de $1 \mathrm{ml}$ con aguja de $25 \mathrm{G} \times 12 \mathrm{ml}$ para plasma, suero y control negativo con solución salina estéril al o,9 \%. Sin dejar espacio muerto, se aplicaron $0,05 \mathrm{ml}$ de suero fresco intradérmico sin diluir, permitiendo la formación de una pequeña "pápula” palpable. Se formó una pápula de 5 a $6 \mathrm{ml}$ de diámetro con algunas variaciones en personas de edad avanzada.

Se llevó a cabo el mismo procedimiento con el plasma 


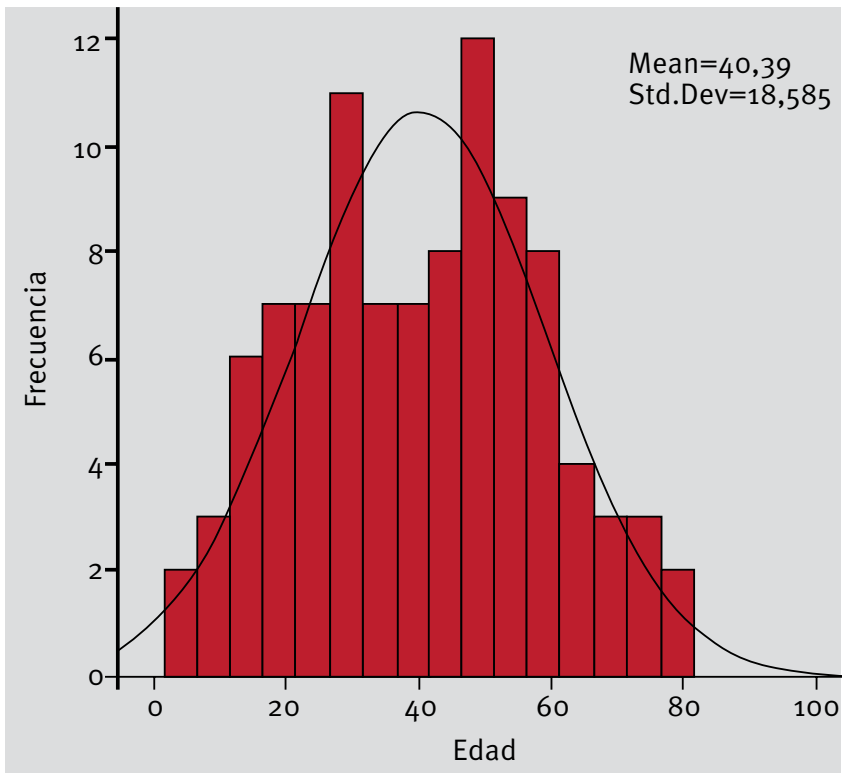

FIGURA 1. Histograma de frecuencias para la edad de los pacientes con urticaria espontánea crónica. Servicio de Alergología Clínica, IPS universitaria -Universidad de Antioquia, Sede Ambulatoria, Medellín, 2006-2011.

del paciente y con el control negativo. El control positivo se hace mediante la administración de histamina por prueba epidérmica por puntura.

La prueba se leyó a los 30 minutos, considerándose positiva cuando el diámetro de la pápula era mayor que el del control negativo por $1,5 \mathrm{~mm}$ o más y, negativa, si habiendo tenido una respuesta positiva a la histamina (formación de un habón de $3 \mathrm{~mm}$ o más), la diferencia entre el tamaño del habón para el plasma o el suero con respecto al control negativo era menor de $1,5 \mathrm{~mm}^{18}$.

\section{Aspectos éticos}

Nuestro estudio, por ser realizado en seres humanos, se basa en el respeto por las personas, la beneficencia, la no maleficencia y la justicia. En este estudio no se solicitó consentimiento informado a los participantes pues, a pesar de ser una cohorte, esta es retrospectiva y basada en la revisión del historial médico registrado en el sistema GHIPS de la IPS Universitaria de la Universidad de Antioquia.

Los pacientes no fueron sometidos a ninguna consulta médica adicional, destinada para esta investigación, pero sí asistieron a los controles médicos programados según el criterio de su médico tratante, especialista en Alergología Clínica. Algunos de ellos ya se encuentran libres de síntomas y, por lo tanto, no acuden personalmente al Servicio de Alergología Clínica de la IPS Universitaria.

Se garantiza la confidencialidad de la información y no se utilizan datos personales identificables en la publicación de los resultados. Los datos de identificación personal permanecen en los archivos utilizados para el estudio, ya que nos permiten identificar las historias clínicas por evaluar, pero en ningún caso serán divulgados, ni pondrán en desventaja a los pacientes participantes en el estudio.

Declaramos que no existe ninguna incompatibilidad de intereses entre los investigadores y los asesores de este estudio.

El resultado primario de este estudio fue explorar la relación entre determinados parámetros clínicos y de laboratorio (edad, sexo, actividad de la enfermedad, presencia de angioedema, coexistencia con otros tipos de urticaria, resultado de la prueba de plasma y suero autólogo, autoinmunidad tiroidea, presencia de otras enfermedades atópicas, necesidad de medicamento y presencia de hipertensión arterial) con la duración de la urticaria crónica.

\section{Análisis estadístico}

Se hizo un análisis descriptivo utilizando distribuciones de frecuencia para las variables cualitativas y, para la variable edad, se indagó sobre la distribución con la prueba de Shapiro-Wilks, encontrándose que era de distribución normal; por lo tanto, se utilizaron la media y la desviación estándar como medidas resumen. Además, se hizo un análisis bivariado exploratorio para identificar posibles factores asociados a la duración de la urticaria crónica, para lo cual se utilizó la prueba t de Student para la edad, la prueba de ji al cuadrado de independencia, cuando la variable independiente era cualitativa, y la prueba exacta de Fisher si tenía valores esperados menores de cinco. En este análisis se calcularon los odss ratios ${ }^{13}$ como medida de asociación.

\section{Resultados}

Se identificaron 99 pacientes con diagnóstico de urticaria crónica, de los cuales, 67 eran mujeres y 32 hombres; el promedio de edad fue de 40,3 $\pm 18,6$ años, con variaciones entre 4 y 79 años (FIGURA 1).

La hipertensión arterial fue la enfermedad concomitante de mayor prevalencia en la población estudiada, seguida del angioedema. Más de la mitad de los pacientes no presentó asociación con otro tipo de urticaria, pero en un poco más del $40 \%$ se asoció con urticaria física y, en cerca del $4 \%$, con urticarias de otros tipos. De los 99 pacientes, alrededor del $30 \%$ presentaban además otras enfermedades alérgicas, de las cuales, las respiratorias fueron las más frecuentes (TABLA 1).

$\mathrm{El} 71,7 \%$ de los pacientes presentó una prueba positiva de plasma y suero autólogo, y el 17,2 \% presentó altera- 


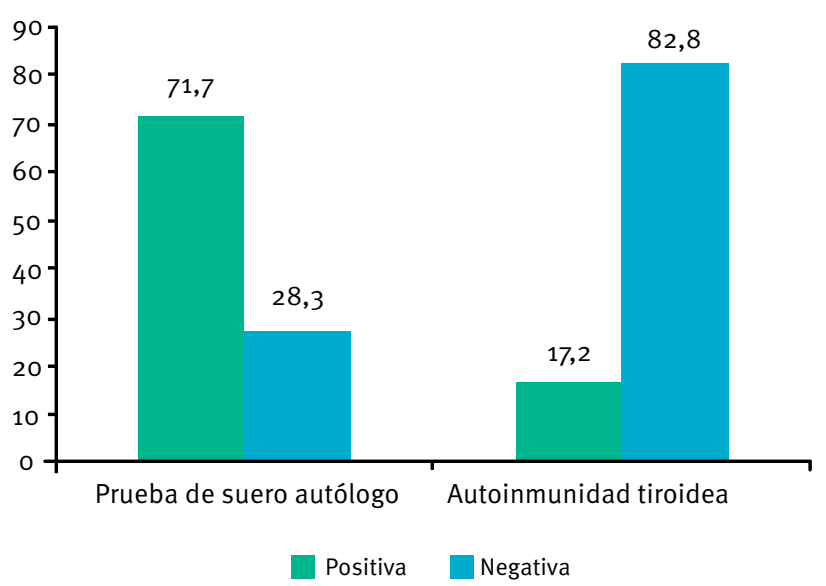

FIGURA 1. Histograma de frecuencias para la edad de los pacientes con urticaria espontánea crónica. Servicio de Alergología Clínica, IPS universitaria -Universidad de Antioquia, Sede Ambulatoria, Medellín, 2006-2011.

\begin{tabular}{|c|c|c|}
\hline \multicolumn{2}{|c|}{ Características clínicas de los pacientes } & n (\%) \\
\hline \multirow{2}{*}{$\begin{array}{l}\text { Hipertensión arterial } \\
\text { asociada }\end{array}$} & No & $72(72,7)$ \\
\hline & Sí & $27(27,3)$ \\
\hline \multirow{2}{*}{$\begin{array}{l}\text { Presencia de } \\
\text { angioedema }\end{array}$} & Sí & $59(59,6)$ \\
\hline & No & $40(40,4)$ \\
\hline \multirow{3}{*}{$\begin{array}{l}\text { Asociación con otros } \\
\text { tipos de urticaria }\end{array}$} & Ausente & $54(54,5)$ \\
\hline & Física & $41(41,4)$ \\
\hline & Otros tipos de urticaria & $4(4,0)$ \\
\hline \multirow{3}{*}{$\begin{array}{l}\text { Otras enfermedades } \\
\text { alérgicas }\end{array}$} & Cutánea & $4(4,0)$ \\
\hline & Respiratorias & $25(25,3)$ \\
\hline & Ausentes & $70(70,7 \%)$ \\
\hline
\end{tabular}

TABLA 1. Características clínicas de los pacientes con urticaria espontánea crónica. Servicio de Alergología Clínica, IPS universitaria - Universidad de Antioquia Sede Ambulatoria, Medellín, 2006-2011.
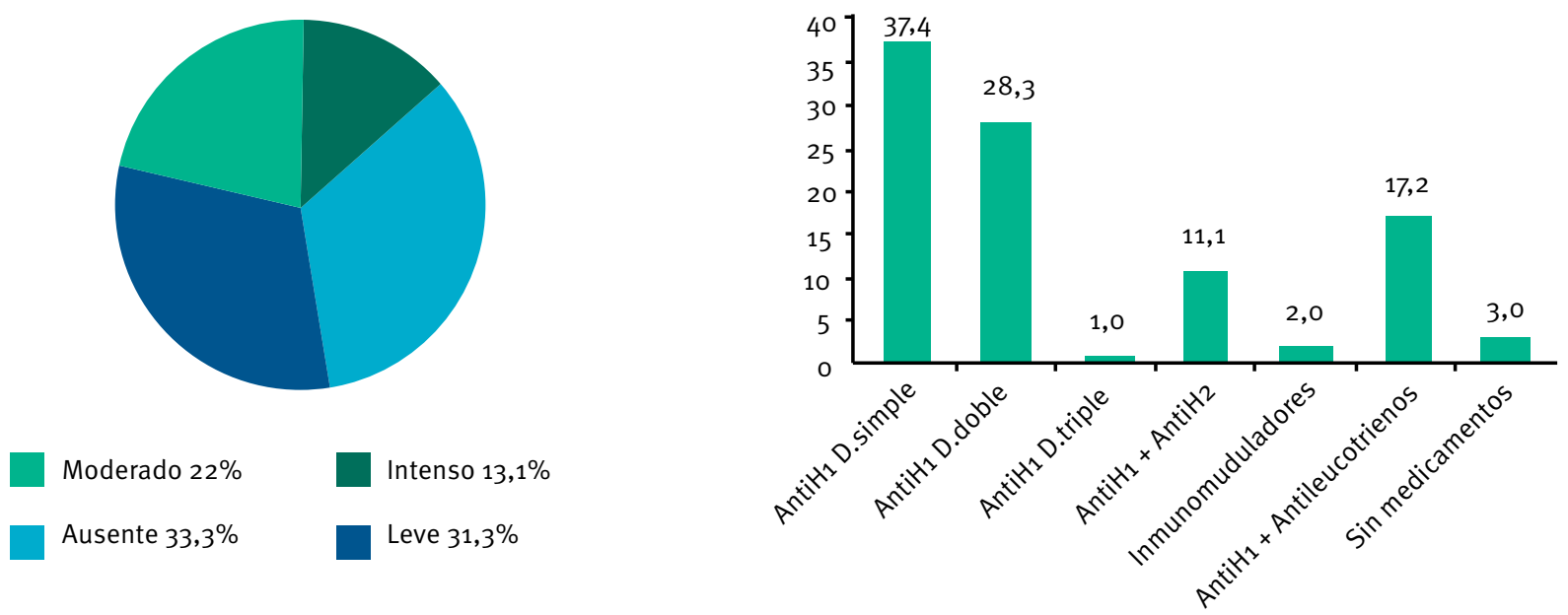

FIGURA 3. Estado actual de la enfermedad y necesidad de medicamentos en los pacientes con urticaria espontánea crónica. Servicio de Alergología Clínica, IPS universitaria - Universidad de Antioquia - Sede Ambulatoria, Medellín, 2006-2011.

ción tiroidea determinada por la presencia de anticuerpos antimicrosómicos, antitiroglobulina positivos o ambos, o prueba de TSH anormal (teniendo en cuenta los valores de referencia de cada laboratorio) (FIGURA 2).

Con respecto a la actividad de la urticaria, el 13,1\% presentaba una actividad intensa; el 21,2 \%, moderada; el 29,3\%, leve, y el 31,3\%, ausente. El 37,4 \% recibía tratamiento con antihistamínicos $\mathrm{H} 1$ a dosis simple $\mathrm{y}$, el 28,3 \%, a dosis doble; el 17,2 \% recibía antihistamínico H1 más antagonista del receptor de leucotrienos; el $11,1 \%$ recibía antihistamínicos $\mathrm{H} 1$ más antihistamínicos
$\mathrm{H} 2$, y solo el $2 \%$ recibía tratamiento con medicamento inmunomodulador (FIGURA 3).

Se compararon las variables clínicas y sociodemográficas en relación con la duración de la urticaria crónica, en dos grupos, duración menor de 60 meses y mayor de 60 meses, y se encontró, aunque sin diferencias estadísticamente significativas, que el sexo femenino tenía una mayor proporción de pacientes con duración mayor de 60 meses, de igual manera que los pacientes con angioedema, otras urticarias y otras enfermedades alérgicas que comprometieran la piel (FIGURA 4). 


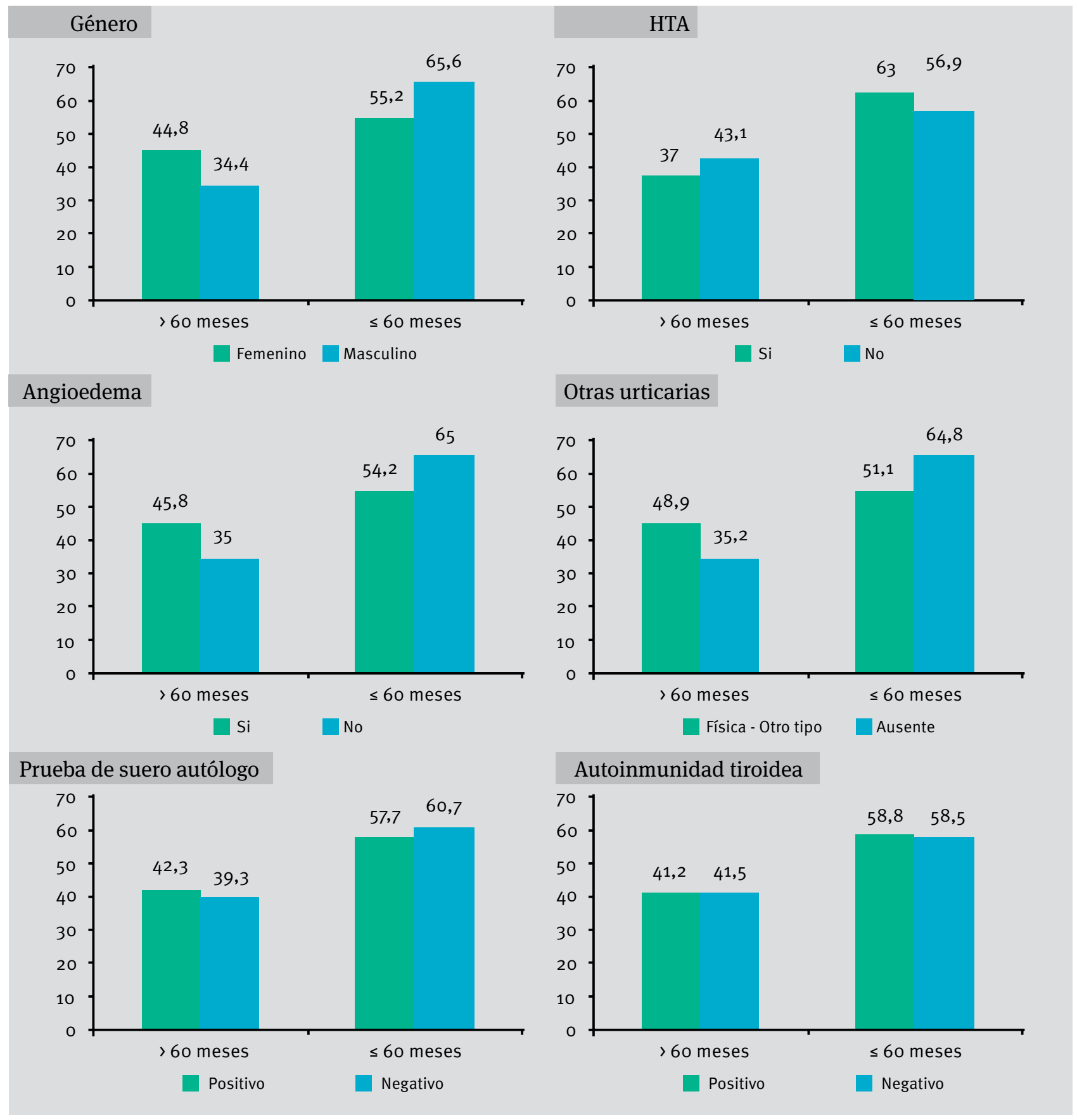

FIGURA 4. Relación de las variables sociodemográfica y clínicas con la duración de la urticaria espontánea crónica. Servicio de Alergología Clínica, IPS universitaria - Universidad de Antioquia - Sede Ambulatoria, Medellín, 2006-2011.

\section{Discusión}

En este estudio se exploran los factores asociados a la duración de la urticaria crónica, en una cohorte de pacientes que asistieron a un servicio de alergología clínica. Es un estudio de naturaleza retrospectiva en el que se obtuvo la información clínica y paraclínica necesaria, extraída de la historia clínica o por llamado telefónico de 99 pacientes.
Nuestra cohorte mostró que la urticaria crónica se presenta en un amplio espectro de edades, distribuido entre los 4 años y los 79 años de edad, con un promedio de 40,3 años, un pico de presentación entre los 20 y 40 años, y un predominio en el sexo femenino, muy si-

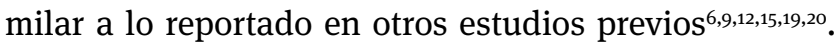
Además, en este grupo de pacientes se vio un segundo pico de presentación entre los 40 y los 60 años, el cual no ha sido descrito en otros estudios. 
Se encontró que el 59,6 \% de los pacientes estudiados presentaba angioedema, que fue ligeramente mayor a lo encontrado en otros estudios con cifras que varían desde el $27 \%$ hasta el $67 \%$ 5,21,22. Con respecto a la asociación con otros tipos de urticaria, el 45,4 \% la presentaba; en la literatura científica se han reportado prevalencias de $10 \%$ hasta $60 \%$ 6,9,22,23.

Otras enfermedades alérgicas respiratorias o cutáneas, como asma, rinitis, conjuntivitis alérgica y dermatitis atópica, se encontraron en el 29,3 \% de los pacientes, similar a lo informado por Kulthanan $(31,8 \%)^{24}$. En otros estudios se ha encontrado historia personal de atopia en $24 \%{ }^{22}$ y otros han reportado asociación hasta de $40 \%{ }^{6}$.

La asociación de urticaria crónica con autoinmunidad tiroidea en nuestro grupo fue de 17,2 \%; esta asociación se ha reportado en diferentes estudios, tan alta como de $30 \%{ }^{25}$, o tan baja como de $2,4 \%{ }^{15}$; sin embargo, en la mayoría de estudios esta asociación se encuentra entre el 12 y el $20 \%{ }^{25-27}$.

El 66,7\% de los pacientes recibía tratamiento para su control únicamente con antihistamínicos de tipo $\mathrm{H} 1$, similar a lo reportado en la literatura científica, donde se describe que más del $50 \%$ de los que reciben antihistamínicos $\mathrm{H} 1$ logran controlar su enfermedad ${ }^{1}$.

Con relación a la duración de la urticaria mayor o menor de 60 meses, se encontró que el porcentaje en el sexo femenino fue superior al porcentaje en el sexo masculino, con una diferencia de 10,8 \%, para una duración mayor de 60 meses, la cual aunque no es estadísticamente significativa, posiblemente debido al tamaño de la muestra, permite identificar una tendencia que en una muestra de mayor tamaño pudiera alcanzar significancia estadística. Sahiner, et al., reportaron un hallazgo similar: en el análisis univariado, ser mujer y mayor de 10 años predecía un pronóstico no favorable ${ }^{28}$.

La presencia de angioedema con respecto a una duración mayor de 60 meses, mostró una diferencia superior al $10 \%$, que no fue estadísticamente significativa posiblemente por el tamaño de la muestra. Esta observación se ha encontrado en otros estudios, en los cuales sí ha tenido significancia estadística, Toubi, et al., encontraron que, en el seguimiento a cinco años, el $45 \%$ de los pacientes con urticaria crónica más angioedema seguían presentando lesiones en la piel, en comparación con el $12 \%$ de aquellos con urticaria crónica sin angioedema $(p=0,002)^{12}$. En otros estudios, sin embargo, no se ha encontrado dicha asociación ${ }^{19,20}$.

La asociación de urticaria crónica con otros tipos de urticarias físicas, es un factor de peor pronóstico que ya se ha demostrado en otros estudios ${ }^{6,19}$. En nuestra cohorte se evidenció que los pacientes con otros tipos de urticarias tienen una diferencia de más del $10 \%$ para una duración mayor de 60 meses, con respecto a los que únicamente presentan urticaria crónica.

En un estudio prospectivo de pacientes remitidos al Servicio de Dermatología de un hospital de tercer nivel de la Universidad de Atenas, se evaluaron 2.523 pacientes con urticaria y, mediante un modelo de análisis de regresión múltiple, se encontró que las variables sexo femenino, presencia de angioedema y de otros tipos de urticarias físicas se asociaban con un peor pronóstico de la urticaria, hallazgos similares a lo encontrado en nuestra población ${ }^{29}$.

No se encontró ninguna asociación entre la duración de la urticaria espontánea crónica con respecto al resultado de la prueba de plasma y suero autólogo. Existe controversia de la utilidad de esta prueba como factor pronóstico de intensidad o duración de la urticaria crónica. En un estudio de 165 pacientes con urticaria crónica, no se encontró ninguna diferencia en cuanto a la duración e intensidad de la enfermedad con respecto al resultado de la prueba de suero autólogo, pero sí un predominio de la prueba positiva en mujeres frente a hombres; además, la frecuencia de los episodios de urticaria crónica era mayor (más de cinco veces por semana) y los títulos de anticuerpos antiperoxidasa eran más altos en los que tenían una prueba positiva ${ }^{30}$. Otros autores también han encontrado asociación entre la intensidad y la duración de la urticaria crónica con respecto a la prueba positiva de suero autólogo ${ }^{12,31}$ o una mayor necesidad de medicamentos antihistamínicos para lograr el control de los síntomas en aquellos con resultado positivos de la prueba ${ }^{32}$. Asimismo, se han reportado pruebas positivas de suero autólogo en pacientes con enfermedades respiratorias altas sin urticaria crónica hasta en 29,8\%, frente a 53,1 \% en pacientes con urticaria crónica ${ }^{33}$. No obstante, en otros estudios no se ha encontrado ninguna asociación entre el resultado de la prueba de suero autólogo y la intensidad o duración de la enfermedad ${ }^{20,34}$.

En nuestro estudio realiza no solo se practió la prueba de suero autólogo, sino también, la de plasma autólogo. La implementación de esta última ha sido tema de mucha controversia en diferentes grupos científicos ${ }^{35-37}$; algunos de ellos argumentan una mayor sensibilidad en el uso de la prueba de plasma autólogo para detectar autorreacción ${ }^{38}$, mientras que otros señalan un posible aumento de los falsos positivos, posiblemente debido al efecto del citrato de sodio, que amerita usar un control negativo de solución salina diluida en este ${ }^{39}$; otros no encuentran ningún beneficio adicional con respecto a sensibilidad y especificidad con la prueba de plasma autólogo, por lo cual no recomiendan su uso adicional a la prueba con suero autólogo ${ }^{37,39}$. Actualmente, la EAACI en conjunto con GA2LEN, recomiendan únicamente el uso de la prueba de suero autólogo para la determinación 
de autorreacción en pacientes con urticaria espontánea crónica, la cual tiene una sensibilidad estimada entre el $44,4 \%$ y el $100 \%$, y una especificidad entre el $27,2 \%$ y el $86,1 \%{ }^{18}$.

Con respecto a la presencia de hipertensión arterial, se encontró que en nuestra cohorte no guardó relación con la duración de la urticaria, a diferencia de lo observado por Nebiolo, et al., en su cohorte prospectiva de 228 pacientes, en quienes encontraron asociación entre la presencia de hipertensión arterial (hazard ratio $=0,71 ; \mathrm{IC}_{95 \%}$ $0,53-0,95 ; p=0,02)$ y la duración de la urticaria, y descartaron asociación entre la duración de la urticaria crónica y la presencia de angioedema (hazard ratio $=0,96$; IC $_{95 \%}$ $0,86-1,08 ; p=0,54]$, resultado de la prueba de suero autólogo (hazard ratio=0.99; $\mathrm{IC}_{95 \%}$ 0,99-1,01; $\mathrm{p}=0,93$ ], presencia de anticuerpos antitiroideos (hazard ratio $=1,00$; IC $_{95 \%}$ 0,99-1,00; $\left.\mathrm{p}=0,495\right]$, anticuerpos antinucleares (hazard ratio $=0,99 ; \mathrm{IC}_{95 \%} 0,99-1,01 ; \mathrm{p}=0,90$ ] y atopia (hazard ratio $\left.=1,12 ; \mathrm{IC}_{95 \%} 0,44-3,28 ; \mathrm{p}=0,72\right]$. Este estudio es el único reportado en la literatura científica en el que se encuentra asociación entre la duración de la urticaria crónica y la presencia de hipertensión arterial ${ }^{20}$.

\section{Conclusión}

En nuestra población, al igual que en otras cohortes descritas previamente, la urticaria crónica es más común en mujeres y afecta especialmente la población en edad productiva con una distribución en todas las edades. La presencia de angioedema fue similar a lo reportado en estudios previos, al igual que la asociación con otros tipos de urticaria, en especial la urticaria física. En la exploración de los factores asociados a la duración, aunque ninguna de las variables estudiadas tuvo significancia clínica, sí se observaron diferencias mayores del $10 \%$ para variables tales como el sexo femenino, la presencia de angioedema y la asociación con otros tipos de urticaria, con relación a una mayor duración de la enfermedad, lo cual concuerda con lo que se ha descrito previamente en la literatura científica y, posiblemente, si se le da continuidad a este estudio y se incluye una mayor muestra, se pueda obtener significancia estadística para estas variables.

La prueba de plasma y suero autólogo en nuestra cohorte no demostró tener ninguna asociación con la duración de la enfermedad, y la utilidad de esta prueba es un tema de controversia. Consideramos, por ahora, que a pesar de la polémica con respecto a esta prueba, debemos seguir las recomendaciones del consenso de expertos publicado por la EAACI y GA2LEN en el 2009 sobre el uso de la prueba de suero autólogo en la determinación de autorreacción en pacientes con urticaria crónica.

\section{Referencias}

1. Maurer M, Weller K, Bindslev-Jensen C, Giménez-Arnau A, Bousquet PJ, Bousquet J, et al. Unmet clinical needs in chronic spontaneous urticaria. A GALEN task force report. Allergy. 2011;66:317-30.

2. Zuberbier T, Asero R, Bindslev-Jensen C, Canonica W, Church MK, Giménez-Arnau A, et al. EAACI/GA(2)LEN/EDF/WAO guideline: Definition, classification and diagnosis of urticaria. Allergy. 2009;64:1417-26.

3. Amar SM, Dreskin SC. Urticaria. Prim Care. 2008;35:141-57.

4. Gaig P, Olona M, Muñoz D, Caballero MT, Domínguez FJ, Echechipia S, et al. Epidemiology of urticaria in Spain. J Investig Allergol Clin Immunol. 2004;14:214-20.

5. Zuberbier T, Balke M, Worm M, Edenharter G, Maurer M. Epidemiology of urticaria: A representative cross-sectional population survey. Clin Exp Dermatol. 2010;35:869-73.

6. Kozel MM, Mekkes JR, Bossuyt PM, Bos JD. Natural course of physical and chronic urticaria and angioedema in 220 patients. J Am Acad Dermatol. 2001;45:387-91.

7. Quaranta JH, Rohr AS, Rachelefsky GS, Siegel SC, Katz RM, Spector SL, et al. The natural history and response to therapy of chronic urticaria and angioedema. Ann Allergy. 1989;62:421-4.

8. Kulthanan K, Jiamton S, Thumpimukvatana N, Pinkaew S Chronic idiopathic urticaria: Prevalence and clinical course. J Dermatol. 2007;34:294-301

9. Humphreys F, Hunter JA. The characteristics of urticaria in 390 patients. Br J Dermatol. 1998;138:635-8.

10. Grob JJ, Gaudy-Marqueste C. Urticaria and quality of life. Clin Rev Allergy Immunol. 2006;30:47-51.

11. O'Donnell BF, Lawlor F, Simpson J, Morgan M, Greaves MW. The impact of chronic urticaria on the quality of life. Br J Dermatol. 1997;136:197-201.

12. Toubi E, Kessel A, Avshovich N, Bamberger E, Sabo E, Nusem D, et al. Clinical and laboratory parameters in predicting chronic urticaria duration: A prospective study of 139 patients. Allergy. 2004;59:869-73.

13. Sabroe RA, Seed PT, Francis DM, Barr RM, Black AK, Greaves MW. Chronic idiopathic urticaria: Comparison of the clinical features of patients with and without anti-FcepsilonRI or anti-IgE autoantibodies. J Am Acad Dermatol. 1999;40:443-50.

14. Caproni M, Volpi W, Giomi B, Cardinali C, Antiga E, Melani L, et al. Chronic idiopathic and chronic autoimmune urticaria: Clinical and immunopathological features of 68 subjects. Acta Derm Venereol. 2004;84:288-90.

15. Silvares MR, Coelho KI, Dalben I, Lastória JC, Abbade LP. Sociodemographic and clinical characteristics, causal factors and evolution of a group of patients with chronic urticaria-angioedema. Sao Paulo Med J. 2007;125:281-5.

16. Zuberbier T, Bindslev-Jensen C, Canonica W, Grattan CE, Greaves MW, Henz BM, et al. EAACI/GA2LEN/EDF guideline: Definition, classification and diagnosis of urticaria. Allergy. 2006;61:316-20.

17. Wedi B, Raap U, Wieczorek D, Kapp A. Urticaria and infections. Allergy Asthma Clin Immunol. 2009;5:10.

18. Konstantinou GN, Asero R, Maurer M, Sabroe RA, Schmid-Grendelmeier P, Grattan CE. EAACI/GA(2)LEN task force consensus report: The autologous serum skin test in urticaria. Allergy. 2009;64:1256-68. 
19. van der Valk PG, Moret G, Kiemeney LA. The natural history of chronic urticaria and angioedema in patients visiting a tertiary referral centre. Br J Dermatol. 2002;146:110-3.

20. Nebiolo F, Bergia R, Bommarito L, Bugiani M, Heffler E, Carosso A, et al. Effect of arterial hypertension on chronic urticaria duration. Ann Allergy Asthma Immunol. 2009;103:407-10.

21. Juhlin L. Recurrent urticaria: Clinical investigation of 330 patients. Br J Dermatol. 1981;104:369-81.

22. Nettis E, Pannofino A, D’Aprile C, Ferrannini A, Tursi A. Clinical and aetiological aspects in urticaria and angio-oedema. Br J Dermatol. 2003;148:501-6.

23. Tanaka T, Kameyoshi Y, Hide M. Analysis of the prevalence of subtypes of urticaria and angioedema. Arerugi. 2006;55:134-9.

24. Kulthanan K, Jiamton S, Rutnin NO, Insawang M, Pinkaew S. Prevalence and relevance of the positivity of skin prick testing in patients with chronic urticaria. J Dermatol. 2008;35:330-5.

25. Najib U, Bajwa ZH, Ostro MG, Sheikh J. A retrospective review of clinical presentation, thyroid autoimmunity, laboratory characteristics, and therapies used in patients with chronic idiopathic urticaria. Ann Allergy Asthma Immunol. 2009;103:496-501.

26. Palma-Carlos AG, Palma-Carlos ML. Chronic urticaria and thyroid auto-immunity. Eur Ann Allergy Clin Immunol. 2005;37:143-6.

27. Doutre MS. Chronic urticaria and thyroid auto-immunity. Clin Rev Allergy Immunol. 2006;30:31-7.

28. Sahiner UM, Civelek E, Tuncer A, Yavuz ST, Karabulut E, Sackesen $\mathrm{C}$, et al. Chronic urticaria: Etiology and natural course in children. Int Arch Allergy Immunol. 2011;156:224-30.

29. Gregoriou S, Rigopoulos D, Katsambas A, Katsarou A, Papaioannou D, Gkouvi A, et al. Etiologic aspects and prognostic factors of patients with chronic urticaria: Non randomized, prospective, descriptive study. J Cutan Med Surg. 2009;13:198-203.

30. Abd El-Azim M, Abd El-Azim S. Chronic autoimmune urticaria: Frequency and association with immunological markers. J Investig Allergol Clin Immunol. 2011;21:546-50.
31. Alyasin S, Karimi AA, Amiri A, Ehsaei MJ, Ghaffarpasand F. Correlation between clinical findings and results of autologous serum skin test in patients with chronic idiopathic urticaria. Allergy Asthma Clin Immunol. 2011;7(Suppl.2):A10.

32. Staubach P, Onnen K, Vonend A, Metz M, Siebenhaar F, Tschentscher I, et al. Autologous whole blood injections to patients with chronic urticaria and a positive autologous serum skin test: A placebo-controlled trial. Dermatology. 2006;212:150-9.

33. Guttman-Yassky E, Bergman R, Maor C, Mamorsky M, Pollack S, Shahar E. The autologous serum skin test in a cohort of chronic idiopathic urticaria patients compared to respiratory allergy patients and healthy individuals. J Eur Acad Dermatol Venereol. 2007;21:35-9.

34. Nettis E, Dambra P, D’Oronzio L, Cavallo E, Loria MP, Fanelli M, et al. Reactivity to autologous serum skin test and clinical features in chronic idiopathic urticaria. Clin Exp Dermatol. 2002;27:29-31.

35. Asero R, Cugno M, Tedeschi A. Autologous plasma and serum skin test in chronic urticaria. Br J Dermatol. 2011;166(6):1362-3.

36. Asero R, Tedeschi A, Cugno M. Is the autologous plasma skin test in patients with chronic urticaria really useless? J Allergy Clin Immunol. 2009;123:1417.

37. Metz M, Giménez-Arnau A, Borzova E, Grattan CE, Magerl M, Maurer M. Frequency and clinical implications of skin autoreactivity to serum versus plasma in patients with chronic urticaria. J Allergy Clin Immunol. 2009;123:705-6.

38. Asero R, Tedeschi A, Riboldi P, Cugno M. Plasma of patients with chronic urticaria shows signs of thrombin generation, and its intradermal injection causes wheal-and-flare reactions much more frequently than autologous serum. J Allergy Clin Immunol. 2006;117:1113-7.

39. Yildiz H, Karabudak O, DoDan B, Harmanyeri Y. Evaluation of autologous plasma skin test in patients with chronic idiopathic urticaria. Br J Dermatol. 2011;165:1205-9. 